CERN-TH/98-155

hep-ph/9805379

\title{
A Strongly-Interacting Phase of the Minimal Supersymmetric Model
}

\author{
Gian F. Giudice® and Alexander Kusenkoß \\ Theory Division, CERN, CH-1211 Geneva 23, Switzerland
}

\begin{abstract}
We argue that in the minimal supersymmetric extension of the Standard Model with a large trilinear coupling both the fundamental Higgs boson and a bound state of squarks (formed via strong scalar interaction) can have a non-zero VEV. This alters drastically the pattern of electroweak-symmetry breaking and the Higgs phenomenology. In particular, the upper bound on the supersymmetric Higgs-boson mass may be relaxed. Also, the Higgs boson can be produced at hadron colliders through a direct coupling to gluons.
\end{abstract}

CERN-TH/98-155

May 1998

\footnotetext{
${ }^{1}$ On leave of absence from INFN, Sezione di Padova, Italy.

${ }^{2}$ E-mail address: Alexander.Kusenko@cern.ch.
} 


\section{Introduction}

Supersymmetry predicts the existence of numerous scalar fields with multifarious interactions. In particular, the two Higgs doublets of the minimal model with softly-broken supersymmetry can couple to squark and slepton bilinears. These cubic interactions, attractive in nature, can give rise to a spectrum of bound states and resonances that one might hope to discover in the future [1].

In this paper we consider the possibility that a strong trilinear interaction produces bound states of two squarks of the third generation. If tightly bound, such states appear as additional (composite) Higgs bosons in the low-energy effective theory. We will explore the empirical implications of this unusual phase of the minimal supersymmetric standard model (MSSM).

Unlike all other coupling constants in the MSSM interaction Lagrangian, which are restricted by supersymmetry, the dimensionful trilinear scalar couplings arise from supersymmetry breaking. Although calculable in some particular models, these couplings are largely unconstrained from both the theoretical and the empirical standpoints. For the lightest generations of fermions, the approximate chiral symmetry constrains the size of the trilinear terms of the corresponding superpartners. Indeed, the trilinear couplings contribute to quark masses at the one-loop level, and the observed small masses of the first two generations require correspondingly small $A$ terms, barring the possibility of unnatural cancellations.

This argument does not apply to the third generation, since the large fermion masses give a sizable breaking of the corresponding chiral symmetry. However, some important upper bounds on the trilinear couplings come from considering the colorand charge-breaking vacua that may have an energy density lower than that of the standard vacuum. If one requires that the standard vacuum be the global minimum

of the potential, one obtains an upper limit on the trilinear terms [2]. Some more conservative and, hence, weaker bounds [3] allow for the possibility that the standard vacuum be a metastable false vacuum with a lifetime that exceeds the age of the Universe. The analyses of refs. [2, 3] give an upper bound on the trilinear coupling $A_{t}$ 
of the stop sector

$$
\left(A_{t} / y_{t}\right)^{2}+3 \mu^{2}<\beta\left(m_{\tilde{t}_{L}}^{2}+m_{\tilde{t}_{R}}^{2}\right)
$$

where $m_{\tilde{t}_{L}}$ and $m_{\tilde{t}_{R}}$ are the diagonal entries of the stop mass matrix, $y_{t} \approx 1$ is the top Yukawa coupling, and $\beta \approx 7.5$ [3] or 3.0 [2], depending on whether one requires the color- and charge-conserving vacuum to be the global minimum of the potential.

In section 2 we will argue that the bound (11) may not apply when the large $A_{t}$ term leads to the appearance of a tight bound state. We will show that, whenever the trilinear coupling is very large, a tightly bound state with the quantum numbers of the Higgs and a binding energy of the order of the electroweak scale can form. Moreover, we will argue that this state can develop a non-zero vacuum expectation value (VEV), mix with the fundamental Higgs bosons, and alter significantly the pattern of the electroweak symmetry breaking and the ordinary Higgs phenomenology.

This offers the possibility for a new realization of softly-broken supersymmetry at the electroweak energy scale. Here and below, we assume the usual MSSM particle content and interaction Lagrangian. However, we will argue that, in the large $A_{t}$ regime, the theory becomes strongly-interacting, and it chooses an unconventional phase, while its usual ultraviolet behavior is preserved. Appealing features of supersymmetry, such as the natural gauge hierarchy, the gauge coupling unification, and the prospects for a quantum theory of gravity, are rooted in the ultraviolet regime and are unchanged by the soft supersymmetry-breaking trilinear couplings, even if these are as large as several $\mathrm{TeV}$. At the same time, the electroweak physics may be changed drastically by the appearance of a composite Higgs boson through stop condensation, and the predicted Higgs phenomenology has many novel features.

The first important consequence is that the upper limit on the mass of the lightest Higgs boson in the MSSM may be relaxed. The second one is that the Higgs production mechanisms and decay modes are modified by the mixing between the stop bound state and the fundamental Higgs. Both conclusions have significant implications for the experimental search of the Higgs boson. 


\section{The Minimal Supersymmetric Model in the Large $A_{t}$ Regime}

Let us start by considering the trilinear coupling $A_{t}$ between the left-handed $Q_{L}$ and the right-handed $\bar{U}_{R}$ third-generation squarks and the corresponding Higgs boson $H_{2}$

$$
V=A_{t} H_{2} Q_{L} \bar{U}_{R}+\text { h.c. }
$$

For simplicity we assume that $A_{t}$ is the only large trilinear coupling, but our discussion can be generalized to more complicated cases.

The left- and right-handed stops are not mass eigenstates. The mass-squared matrix has the form:

$$
M^{2}=\left(\begin{array}{cc}
\tilde{m}_{L}^{2}+m_{t}^{2} & A_{t}\left\langle H_{2}\right\rangle-\mu m_{t} / \tan \beta \\
A_{t}\left\langle H_{2}\right\rangle-\mu m_{t} / \tan \beta & \tilde{m}_{R}^{2}+m_{t}^{2}
\end{array}\right) .
$$

Here $m_{t}$ is the top-quark mass, $\tilde{m}_{L, R}^{2}$ are the supersymmetry-breaking stop-mass parameters, $\mu$ is the Higgs mixing mass, and $\tan \beta$ is the ratio of Higgs VEVs. We define the stop mixing angle $\theta_{t}$, such that $\tilde{t}_{1}=\cos \theta_{t} \tilde{t}_{L}+\sin \theta_{t} \tilde{t}_{R}$ is the heavier of the two mass eigenstates, and $\tilde{t}_{2}=-\sin \theta_{t} \tilde{t}_{L}+\cos \theta_{t} \tilde{t}_{R}$ is the lighter one.

Since the interaction in eq. (2) provides an attractive force mediated by the exchange of the scalar quanta, we expect that bound states can form if the coupling $A_{t}$ is large enough, and if the exchanged particle is sufficiently light. We consider the case in which the Higgs-boson mass is significantly lower than the stop masses, and we argue that the theory predicts two-stop composite states with binding energies of the order of the electroweak scale.

This speculation is supported by the observation that our theory, in the limit in which we neglect all interactions other than the one in eq. (2), is identical with the Wick-Cutkosky model [4] (for reviews, see ref. [5]). The Bethe-Salpeter equation for the bound state in this model,

$$
\left[\left(\frac{1}{2} M_{B S}+p\right)^{2}+m^{2}\right]\left[\left(\frac{1}{2} M_{B S}-p\right)^{2}+m^{2}\right] \psi(p)=\frac{4 i A^{2}}{(2 \pi)^{4}} \int d^{4} k \frac{\psi(k)}{(p-k)^{2}+m_{H}^{2}},
$$


can be solved analytically in the ladder approximation and in the limit of a masslessparticle exchange $\left(m_{H}=0\right)$. Here $M_{B S}, m$, and $m_{H}$ are, respectively, the masses of the bound state, of the component particle, and of the exchanged particle; and $A$ is a generic trilinear coupling. Using the known solution of eq. (4) [4, 5], we obtain that the bound states of two lighter stops $\tilde{t}_{2}$ have masses

$$
\begin{gathered}
M_{S_{n}}=2 m_{\tilde{t}_{2}} \sqrt{1-\frac{\lambda^{2}}{4 n^{2}}} \quad n=1,2, \ldots \\
\lambda=\frac{1}{16 \pi}\left(\frac{A_{t} \cos \alpha \sin 2 \theta_{t}}{\sqrt{2} m_{\tilde{t}_{2}}}\right)^{2} .
\end{gathered}
$$

Here $\cos \alpha$ measures the $\mathrm{H}_{2}$ component of the lighter Higgs scalar.

The result of eq. (5) has been derived in the ladder approximation, assuming $m_{H}=$ 0. We note that these common approximations suffer from some well-known drawbacks. In particular, the ladder approximation explicitly violates the crossing symmetry, mistreats the relativistic limit in the case of large masses, and, in the case of Yang-Mills theories, is not gauge-invariant. Therefore, although one can use the solution of the BetheSalpeter equation as an indication of the presence of some bound states, one should take eq. (5) with a grain of salt. A recently reported solution to the Bethe-Salpeter equation in the Feshbach-Villars formulation [6], $M_{S_{n}}=2 m_{\tilde{t}_{2}} \sqrt{\left(1+\sqrt{1-\lambda^{2} / n^{2}}\right) / 2}$, gives a larger binding energy than that obtained in the ladder approximation. Concerning the $m_{H}=0$ limit, numerical studies [0] have shown that, in the Wick-Cutkosky model with a massive intermediate particle, the bound state exists as long as the coupling $\lambda$ in eq. (6) is larger than some critical value $\lambda_{c}$. In addition, our case is further complicated by the mixing of the bound state and the fundamental Higgs, as discussed below.

In conclusion, the analyses of the Bethe-Salpeter equation support our speculation that there exists a region in the parameter space of the MSSM in which two stops form a bound state with binding energy of the order of the electroweak scale. This occurs for values of the coupling $\lambda$ of order unity, where the theory becomes strongly interacting. In terms of the supersymmetry-breaking parameters, this happens for large values of $A_{t}$ (say of the order of a few $\mathrm{TeV}$ ) and considerably smaller values of 
$\tilde{t}_{2}$ (of the order of a few hundred $\mathrm{GeV}$ ). This mass hierarchy can be the result of an approximate cancellation in the determinant of the stop mass matrix in eq. (3), which indeed occurs in the large $A_{t}$ regime.

Although lacking a rigorous proof, this description of the large $A_{t}$ phase appears quite plausible to us. We hope that future studies will give further support to this conclusion. Since the relevant degrees of freedom in the problem are scalar fields, a lattice approach appears a well-suited tool for analyzing the dynamical properties of this theory.

From a phenomenological point of view, the lightest $s$-wave bound state, denoted here by $S$, plays an important role. We expect that $S$ is a color-singlet $s$-wave combination of $\left[\tilde{t}_{2}^{\star} \tilde{t}_{2}\right]$. The theory also contains a series of resonances with different Standard Model (SM) quantum numbers, e.g., the color-octet combination of $\left[\tilde{t}_{2}^{\star} \tilde{t}_{2}\right]$, and the charge- $4 / 3$ color sextuplet or antitriplet $\left[\tilde{t}_{2} \tilde{t}_{2}\right]$. Depending on the supersymmetric parameters, bound states involving $\tilde{t}_{1}$ or the sbottom may also be formed. For instance, consider the state $S^{\prime}=\left[\tilde{t}_{2}^{\star} \tilde{t}_{1}\right]$. Solving the corresponding Bethe-Salpeter equation in the ladder approximation and in the limit of massless-particle exchange, we find the analogue of eq. (5) for the lowest-lying bound-state mass

$$
M_{S^{\prime}}=\left[\left(m_{\tilde{t}_{1}}+m_{\tilde{t}_{2}}\right)^{2}-\frac{\left(A_{t} \cos \alpha \cos 2 \theta_{t}\right)^{4}}{(32 \pi)^{2} m_{\tilde{t}_{1}} m_{\tilde{t}_{2}}}\right]^{1 / 2}
$$

Likewise, electrically-charged resonances can be formed by the bound states of the sbottom and the stop $\left[\tilde{b}_{2}^{\star} \tilde{t}_{2}\right]$. Unless the sbottom and the charged Higgs (which mediates the interaction) are significantly lighter than the mass scale of the trilinear coupling, the binding energy of the charged resonance should be small.

At sufficiently low energies, the stop fields do not represent the appropriate degrees of freedom in the low-energy effective theory, which must be described in terms of the bound states. We cannot compute perturbatively the effective potential involving the fundamental Higgs doublets and the bound states, and therefore we have to consider the possibility that the system can be in any of the following phases.

(i) $\mathbf{S U}(2) \times \mathbf{U}(1)$-symmetric phase, in which all scalars have zero VEVs. 
(ii) Ordinary Higgs phase, in which the two Higgs doublets have non-zero VEVs, but both the stop fields and the $s$-wave bound states have zero VEVs, $\langle\tilde{t}\rangle=\langle S\rangle=0$. This is the usual (color- and charge-preserving) vacuum.

(iii) Color- and charge-breaking vacuum with $\langle\tilde{t}\rangle \neq 0$. This phase is possible if the $A_{t}$ coupling is sufficiently large. However, this is not the only possibility in the limit of strong trilinear coupling. In addition, there may exist a

(iv) composite Higgs phase, in which the two fundamental Higgs bosons and the bound state $S$ have non-zero VEVs, although $\left\langle\tilde{t}_{1}\right\rangle=\left\langle\tilde{t}_{2}\right\rangle=0$. In this case $S$ plays the role of an additional Higgs field, contributing to the electroweak breaking.

The upper bound (四) on the value of the trilinear coupling was derived under an implicit assumption that, as the coupling $A_{t}$ increases, the system remains in phase (iii). This is not necessarily the case. If a transition to phase (iv) takes place at some large value of $A_{t}$, then the bound (1) does not apply. It is beyond the scope of our analysis to determine the phase diagram of the theory. A calculation on a lattice is probably the only reliable tool for answering this question, which depends essentially on the non-perturbative physics. Here we just assume that, in a certain range of parameters, the system chooses phase (iv), and we will investigate the theoretical and experimental consequences of this assumption.

It appears quite plausible that $S$ can develop a non-zero VEV when $S U(2) \times U(1)$ is spontaneously broken by the Higgs field. Indeed, as we will discuss in the next section, eq. (2) implies a mixing mass term between $S$ and $H_{2}$. A non-zero VEV for $\mathrm{H}_{2}$ generates a tadpole term that triggers a non-zero $\langle S\rangle$.

In phase (iv), $S$ participates in the electroweak breaking and therefore behaves as a third Higgs boson. Since the overall size of electroweak breaking is fixed by the $W$ and $Z$ masses, the relation between the Yukawa couplings and the known fermion masses changes. Moreover, the top-quark mass receives new contributions from large one-loop corrections involving the stop exchange and the $A_{t}$ coupling, and also from a tree-level chirality-violating effective coupling between the top quark and $S$, after integrating out the gluino. As a result, the running top-quark Yukawa is smaller than 
that usually predicted, and very low values of $\tan \beta$ are consistent with the absence of a Landau pole up to very high energies. However, the effective coupling of $S$ to quarks is restricted by the presence of flavour violations mediated by neutral Higgs exchange.

It is interesting to study how the coupling $\lambda$ in eq. (6) evolves with the renormalization scale. The corresponding RG equation depends on several unknown supersymmetrybreaking parameters. However, it is easy to show that there exists a range of parameters in which the $A_{t}$ coefficient grows at low energies faster than $\tilde{m}_{L, R}$, because of the gluino mass effect. Under these conditions, the coupling $\lambda$ remains small down to some lowenergy scale at which an approximate cancellation takes place in the determinant of the mass matrix in eq. (3). Then $m_{\tilde{t}_{2}}$ becomes significantly lower than the typical scale of $\tilde{m}_{L, R}$. This triggers a rapid growth of $\lambda$ to some large, non-perturbative values. In this way it is possible to have a situation in which the high-energy behavior of the theory is analogous to the ordinary MSSM behavior, with the stop fields describing dynamical degrees of freedom. Non-perturbative effects arise only at energies of the order of the electroweak scale, where at least one of the stops is confined in a bound state. In particular, this means that the prediction of gauge-coupling unification remains unaffected. The non-perturbative $A_{t}$ interactions give an effective contribution that can be absorbed in the unknown low-energy threshold corrections. On the other hand, because of the new contributions to the top-quark mass, this theory does not reproduce the usual perturbative results for the extrapolation of the third-generation Yukawa couplings to high energies.

\section{Phenomenological Implications}

In the previous section we argued that there exists a parameter range in which the $s$-wave color-singlet bound state $S=\left[\tilde{t}_{2}^{\star} \tilde{t}_{2}\right]$ develops a VEV. Under the same circumstances, we also expect that $S$ mixes with the fundamental Higgs scalars. Let us consider the low-energy effective theory valid at the electroweak scale. It contains the new scalar degree of freedom, namely the composite Higgs boson $S$. The very coupling in eq. (2) that gives rise to $S$ in the low-energy effective theory causes the mixing 
between composite and fundamental Higgs bosons. The effective potential contains a term linear in both $\mathrm{S}$ and $\mathrm{H}_{2}$ :

$$
V_{\text {eff }}=m_{H S}^{2} S H_{2}+\text { h.c. }
$$

Here $m_{H S}^{2}=A_{t} f$, where $A_{t}$ is the trilinear coupling and $f$ is a form factor that depends on the strong dynamics and is of the order of the electroweak scale.

Knowledge of the effective potential requires a non-perturbative calculation. Since there is effectively only one mass scale in the problem (the electroweak scale), it is difficult to speculate on the form of the potential. In general, we expect some polynomial interactions in $S$ and $H_{1,2}$ with couplings (in units of the electroweak scale) of order one. The perturbative predictions of the Higgs mass spectrum are no longer valid. In particular, new Higgs quartic couplings in the effective potential are generated. Therefore, one cannot trust the usual upper bound on the supersymmetric Higgs mass, although we are not able to quantify the non-perturbative contribution. Nevertheless, it is interesting that the absence of a very light Higgs boson does not necessarily imply the demise of low-energy supersymmetry. In fact, if the lightest Higgs boson is heavy, this may signal the strongly-coupled regime of the MSSM with stop condensation.

Let us now turn to the phenomenology at high-energy colliders. In the regime under consideration, the theory predicts various bound states and resonances with different SM quantum numbers, as illustrated in sect. 2. At hadron colliders $\left[\tilde{t}^{\star} \tilde{t}\right]$ states can be singly produced via gluon fusion, while $[\tilde{t} \tilde{t}]$ states, which carry flavour number, must be produced in pairs or accompanied by two top quarks. If it is kinematically possible, heavy resonances will decay through the strongly-coupled scalar dynamics into a pair of lighter Higgses (fundamental or composite). Otherwise, their decay modes are quite dependent on the mass spectrum and the supersymmetry-breaking parameters.

Here we want to concentrate on the lightest state in the scalar sector, $h$. As previously argued, this is a linear combination of the fundamental Higgs bosons and the $s$-wave, color-singlet bound state $S$

$$
|h\rangle=\frac{\cos \phi}{\sqrt{2}}\left(\sin \alpha\left|H_{1}^{0}\right\rangle+\cos \alpha\left|H_{2}^{0}\right\rangle\right)+\sin \phi|S\rangle .
$$


The experimental signatures then depend on the unknown mixing angles, and, in particular, on $\sin \phi$, which sets the fraction of composite Higgs in the lightest scalar. Since the properties of the fundamental Higgs components are well known, we focus here on the properties of the $S$ component of the scalar state $h$.

We note in passing that the kinematics allow $S$ to form a true bound state since its formation time is significantly shorter than its lifetime. Indeed, the formation time is characterized by the inverse of the binding energy, $i$. $e$. the inverse of the electroweak scale. On the other hand, the $S$ decay occurs via perturbative interactions, either QCD in the case of stop-antistop annihilation, or weak interactions in the case of stop decay. Therefore, the $S$ decay width is considerably smaller than the typical electroweak scale. This is in contrast with the case of stoponium bound states previously considered in the literature [8, 9], in which perturbative QCD provided the attractive force, and the binding energy was only about $1 \%$ of its mass.

The $S$ bound state can decay through annihilation of its constituents or via decay of the constituents themselves. In the latter case, the most likely processes are stop decays into charginos or neutralinos, $\tilde{t}_{2} \rightarrow t \chi^{0}$ and $\tilde{t}_{2} \rightarrow b \chi^{+}$, which however can occur only if $m_{h}>m_{\tilde{t}_{2}}+m_{\chi^{0}}+m_{t}$ and $m_{h}>m_{\tilde{t}_{2}}+m_{\chi^{+}}+m_{b}$, respectively. The process $\tilde{t}_{2} \rightarrow c \chi^{0}$ is strongly suppressed by flavor conservation and the decay $\tilde{t}_{2} \rightarrow t \tilde{g}$ is probably inhibited by the large gluino mass. For instance the decay rate into charginos $\chi_{i}^{+}(i=1,2)$ is

$$
\Gamma\left(\tilde{t}_{2} \rightarrow b \chi_{i}^{+}\right)=m_{\tilde{t}_{2}} \frac{g^{2}}{16 \pi}\left(V_{i 1} \sin \theta_{t}+\frac{m_{t} V_{i 2}}{\sqrt{2} m_{W} \sin \beta} \cos \theta_{t}\right)^{2}\left(1-\frac{m_{\chi_{i}^{+}}^{2}}{m_{\tilde{t}_{2}}^{2}}\right)^{2} .
$$

Here $V$ is the usual chargino diagonalization matrix and $\Gamma(S)=2 \Gamma\left(\tilde{t}_{2}\right)$.

The stop annihilation process can lead to a pair of gauge bosons, of charginos, of neutralinos, or to a quark-antiquark pair. The annihilation into gluons is likely to dominate, with a rate given by

$$
\Gamma(S \rightarrow g g)=\frac{8}{3} \alpha_{s}^{2} \Delta
$$

\footnotetext{
${ }^{3}$ Complete formulae for stop and stoponium decay rates can be found in ref. [9].
} 
In the non-relativistic limit, $\Delta$ is given in terms of the $S$-wavefunction at the origin $R(0)$ by the expression $\Delta=|R(0)|^{2} / M_{S}^{2}$. In our case, the bound state is relativistic, as the binding energy is of the order of the particle masses. Nevertheless, we can still parametrize the decay rate into gluons by eq. (11), taking $\Delta$ as an unknown parameter of the order of a few hundred GeV.

Because of the QCD charge of its constituents, the $S$ component of $h$ has a direct coupling to gluons, which can significantly increase the Higgs production rate at hadron colliders. In the narrow-width approximation, the production cross section is

$$
\sigma(p p \rightarrow h+X)=\frac{\pi^{2}}{8 m_{h}^{3}} \sin ^{2} \phi \Gamma(S \rightarrow g g) \int_{\tau}^{1} \frac{d x}{x} \tau G\left(x, Q^{2}\right) G\left(\tau / x, Q^{2}\right),
$$

where $\tau=m_{h}^{2} / s, \Gamma(S \rightarrow g g)$ is given in eq. (11) and $G$ describes the gluon density. The cross section at $p \bar{p}$ colliders is the same, since the production occurs via gluon fusion. Although the $S$ width is typically several $\mathrm{GeV}$, the narrow-width approximation is not necessarily inadequate since the experimental dijet mass resolution is about $10 \%$. The numerical values of the total cross sections (in units of $\left.(\Delta / 100 \mathrm{GeV}) \sin ^{2} \phi\right)$ for the Tevatron and the LHC are shown in fig. 1, using $Q^{2}=m_{h}^{2}$.

The experimental signature of the composite Higgs depends on the decay modes. If $\Delta \simeq M_{S}$, then the two-gluon decay rate in eq. (11) is larger than the one in eq. (10). Nevertheless, depending on the supersymmetry-breaking terms and the nonperturbative parameter $\Delta$, it is possible that the mode in eq. (10) or other decay modes into supersymmetric particles become the dominant channel. An experimental search should consider these various possibilities.

When the decay into two gluons dominates, the composite Higgs appears as a peak in the invariant-mass distribution of the dijet cross section. CDF has searched for anomalous resonances in the dijet system and derived limits on the corresponding production cross-section [10]. Unfortunately, the present limits are too weak to set meaningful bounds on particles produced from gluon fusion, such as the composite Higgs $S$. A more promising way of searching for $S$ is to employ its decay into two photons, since the background can be effectively reduced by simple acceptance cuts. 


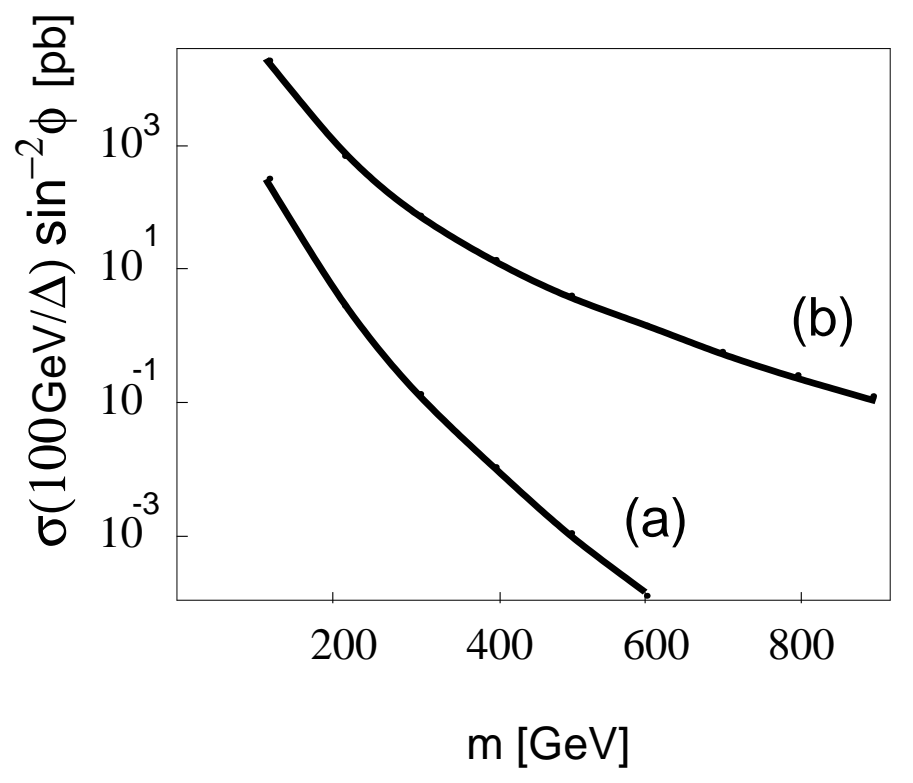

Figure 1: Production cross-section for the scalar state $h$ in units of $\Delta\left(\sin ^{2} \phi\right) / 100 \mathrm{GeV}$, as a function of the $h$ mass. Curve (a) refers to the Tevatron $(\sqrt{s}=1.8 \mathrm{TeV})$ and curve (b) to the $\operatorname{LHC}(\sqrt{s}=14 \mathrm{TeV})$.

Assuming that the non-perturbative form factor $\Delta$ is the same in the decay into gluons and photons, we find

$$
\Gamma(S \rightarrow \gamma \gamma)=\frac{8}{9}\left(\frac{\alpha}{\alpha_{s}}\right)^{2} \Gamma(S \rightarrow g g) .
$$

This small branching ratio into two photons can be used as a distinguishing feature of the composite Higgs both at the Tevatron and at the LHC. When the decay into supersymmetric particles dominate, the experimental signature is quite dependent on the model parameters, but in general it leads to a structure of decay chains with many particles in the final state. Leptonic decays of charginos and heavy neutralinos provide the best channel for discovery.

The production cross-section for $S$ at the Tevatron is limited by the small gluon luminosity at $\sqrt{s}=1.8 \mathrm{TeV}$. Heavier resonances produced in quark-antiquark collisions could then provide the discovery mode. A good candidate is the color-octet $p$-wave bound state $P_{8}=\left[\tilde{t}_{2}^{*} \tilde{t}_{2}\right]$. Since $P_{8}$ is likely to have binding energy comparable to its 


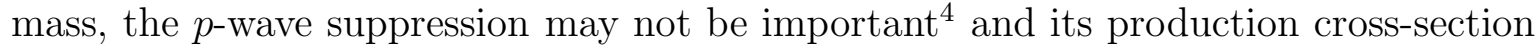
at the Tevatron can be significant. Depending on the masss spectrum, $P_{8}$ can decay into lighter bound states, as in $P_{8} \rightarrow h g$ or $P_{8} \rightarrow h S_{8}$, where $S_{8}$ is the $s$-wave colorsinglet state. Alternatively, it can disintegrate through stop decay. In any case, the final state presents a rich hadronic activity.

\section{Conclusions}

We have argued here that the well-known and well-studied MSSM may manifest itself in a very different way than usually expected. In a possible range of the yet unknown supersymmetry-breaking parameters, the theory becomes strongly interacting. The scalar partners of the top quarks form bound states that can mix with the fundamental Higgs bosons. It appears plausible that the color-singlet $s$-wave bound state of two stops develops a non-zero VEV and takes part in the electroweak symmetry breaking. The Higgs phenomenology of the MSSM can differ drastically from the predictions based on perturbative analyses. In particular, the upper bound on the lightest Higgs boson mass may be relaxed. Moreover, the Higgs boson can be produced at a hadron collider via its bound-state component, which has a direct coupling to two gluons.

This strongly-interacting regime occurs when the trilinear coupling $A_{t}$ becomes large, of the order of a few $\mathrm{TeV}$, and drives the stop mass to values of a few hundred $\mathrm{GeV}$, below the typical scale of the stop supersymmetry-breaking masses. The effective scalar potential at the electroweak scale is dominated by non-perturbative effects, and, therefore, the usual analysis of the color- and charge-breaking minima does not apply. It is quite plausible that the trilinear scalar interaction is weak at high energies and enters its non-perturbative regime only near the electroweak scale. The usual highenergy behavior of the MSSM remains unchanged and, in particular, the predictions for the gauge-coupling unification are unaffected.

We emphasize that the stop condensation and the composite scalar states we hy-

\footnotetext{
${ }^{4}$ For a calculation of the $p$-wave suppression of stoponium production in the non-relativistic limit, see ref. [1].
} 
pothesized are not meant to replace the Higgs sector. They just appear as a dynamical consequence of the ordinary MSSM in the regime of large $A_{t}$. Fundamental Higgs bosons are present in the theory, along with the composite states bound by the strong scalar force. In this respect, our approach is very different from the idea of topcondensate models [12], in which new effective interactions eliminate the need for a fundamental Higgs. However, even when the effective interaction is mediated by a fundamental field [13], one is forced to introduce a large mass scale, which brings back the naturalness problem of the SM Higgs sector. In our case, it is the ultraviolet behavior of softly-broken supersymmetry that is ultimately responsible for protecting the gauge hierarchy.

We have presented a plausible scenario for the dynamical behavior of MSSM in the large $A_{t}$ regime. However, only a truly non-perturbative calculation could prove our speculations and quantify our predictions. We hope that lattice simulations of the theory (which essentially involves only scalar degrees of freedom) will allow one to make further progress in understanding the strong-coupling phase of the MSSM.

We wish to thank G. Altarelli, R. Barbieri, F. Feruglio, M. Mangano, R. Rattazzi, M. Shaposhnikov, and C. Wagner for very helpful discussions.

\section{References}

[1] A. Kusenko, V.A. Kuzmin, and I.I. Tkachev, Phys. Lett. B, in press hepph/9801405).

[2] M. Claudson, L. Hall, and I. Hinchliffe, Nucl. Phys. B228 (1983) 501; L. ÁlvarezGaumé, J. Polchinski, and M. Wise, Nucl. Phys. B221 (1983) 495; J.M. Frère, D.R.T. Jones, and S. Raby, Nucl. Phys. B222 (1983) 11; C. Kounnas, A.B. Lahanas, D.V. Nanopoulos, and M. Quirós, Nucl. Phys. B228 (1983) 501; J.P. Derendinger and C.A. Savoy, Nucl. Phys. B237 (1984) 307; M. Drees, M. Glück, and K. Grassie, Phys. Lett. B157 (1985) 164; J.F. Gunion, H.E. Haber, and M. Sher, Nucl. Phys. B306 (1988) 1; H. Komatsu, Phys. Lett. B215 (1988) 323; 
P. Langacker and N. Polonsky, Phys. Rev. D50 (1994) 2199; A.J. Bordner hepph/9506409); J.A. Casas, A. Lleyda, and C. Muñoz, Nucl. Phys. B471 (1996) 3; S.A. Abel and C.A. Savoy (hep-ph/9803218).

[3] A. Kusenko, P. Langacker, and G. Segrè, Phys. Rev. D 54 (1996) 5824; A. Kusenko and P. Langacker, Phys. Lett. B 391 (1997) 29.

[4] G.C. Wick, Phys. Rev. 96 (1954) 1124; R.E. Cutkosky, Phys. Rev. 96 (1954) 1135.

[5] C. Itzykson and J.-B. Zuber, Quantum Field Theory, McGraw-Hill, New York 1980; N. Nakanishi, Prog. Theor. Phys. Suppl. 95 (1988) 1; Z.K. Silagadze thepph/9803307).

[6] M. Barham and J. Darewych, J. Phys. A 31 (1998) 3481.

[7] E. Zur Linden and H. Mitter, Nuovo Cim. B61 (1969) 389; C.-R. Ji and R.J. Furnstahl, Phys. Lett. B167 (1986) 11; L. Di Leo and J. Darewych, Can. J. Phys. 70 (1992) 412; T. Nieuwenhuis and J.A. Tjon, Phys. Rev. Lett. 77 (1996) 814.

[8] V. Barger and W.-Y. Keung, Phys. Lett. B211 (1988) 355; K. Hagiwara, K. Kato, A.D. Martin, and C.-K. Ng, Nucl. Phys. B344 (1990) 1; H. Inazawa and T. Morii, Phys. Rev. Lett. 70 (1993) 2992.

[9] M. Drees and M.M. Nojiri, Phys. Rev. D49 (1994) 4595.

[10] F. Abe et al. (CDF Coll.), Phys. Rev. D55 (1997) 5263.

[11] I.I. Bigi, V.S. Fadin, and V. Khoze, Nucl. Phys. B377 (1992) 461.

[12] Y. Nambu, in Proc. Int. Workshop on New Trends in Strong Coupling Gauge Theories, Nagoya, 1988, eds. M. Bando, T. Muta, and Y. Yamawaki; W.A. Bardeen, C.T. Hill, and M. Lindner, Phys. Rev. D41 (1990) 1647.

[13] D.E. Clague and G.G. Ross, Nucl. Phys. B364 (1991) 43. 